\title{
A Difference Method for a Singular Boundary Value Problem of Second Order
}

\author{
By Ewa Weinmüller
}

\begin{abstract}
The standard three-point discretization applied to the numerical solution of linear boundary value problems for second order systems with a singularity at the origin is investigated. A number of numerical examples illustrating the theoretical results are presented.
\end{abstract}

1. Introduction. We consider the three-point difference method applied to the linear boundary value problem

$$
\begin{aligned}
y^{\prime \prime}(t)-\frac{A_{1}(t)}{t} y^{\prime}(t)-\frac{A_{0}(t)}{t^{2}} y(t) & =f(t), \quad 0<t \leqslant 1, \\
B_{0} Y(0)+B_{1} Y(1) & =\beta,
\end{aligned}
$$

where $Y(t)=\left(y(t), y^{\prime}(t)\right)^{T}$. Here, $y$ and $f$ are vector-valued functions of dimension $n, A_{0}(t)$ and $A_{1}(t)$ are $n \times n$ matrices, $B_{0}$ and $B_{1}$ are $m \times 2 n$ constant matrices and $\beta$ is an $m$-vector, where $m \leqslant 2 n$.

The numerical solution of scalar equations of this type has been investigated by several authors; see Jamet [5], Natterer [10], Russel and Shampine [15]. The finite difference method discussed here has been applied by Natterer [10] to the scalar problem. Jamet [5] and Russel and Shampine [15] study the problem in a form which often occurs in chemistry or physics, viz., $A_{0}=0$ while $f$ is a function of $t$ and $y$.

First order systems with singularity of the first kind have been discussed by Brabston and Keller [1] and de Hoog and Weiss [2]. Our investigations are based on the techniques developed in [2] and therefore we transform the second order system (1.1a) to the first order one; see (3.2a). In practical applications nonlinear versions of (1.1) also arise; some examples from mechanics and chemistry may be found in Keller and Wolfe [7], Parter, Stein and Stein [12] and Rentrop [14].

It is the main aim of this paper to present the basic ideas of the treatment of second order systems, using the spectral decomposition (proposed in [2]) of the matrix $M \equiv M(0)$, cf. (3.2a), and the contraction arguments. The basic analytic properties of (1.1) like existence, smoothness and uniqueness of solutions have been studied by the author in [16].

The outline of the paper is as follows. In Section 3 we briefly discuss the analytical results for the continuous solutions of (1.1), which we require for the investigations of the numerical scheme in Section 4.

Received December 6, 1982; revised June 11, 1983.

1980 Mathematics Subject Classification. Primary 65L10, 39A10.

(5) 1984 American Mathematical Society $0025-5718 / 84 \$ 1.00+\$ .25$ per page 
Here, as in the analysis of problem (1.1), see [16], we transform the difference equations for (1.1a) to first order difference equations. The order of convergence depends on the smoothness of $y$ and the eigenvalues of $M$. For $f \in C^{2}$, it can be shown that the order of convergence is $h^{q}|\ln h|^{p}, p \geqslant 0$, where $h$ is the equidistant gridspacing on $[0,1]$. If all eigenvalues of $M$ have nonpositive real parts, then $q=2$; otherwise $q$ depends on the smallest positive real part $\sigma_{+}$and $q=\min \left(\sigma_{+}, 2\right)$.

In Section 5, we present a number of numerical experiments illustrating the theory.

2. Notations and Preliminary Results. We use the following notation: $X^{n}$ is the space of complex-valued vectors of dimension $n$, and we use $|\cdot|$ to denote the maximum norm in $X^{n}$,

$$
|x|=\left|\left(x_{1}, x_{2}, \ldots, x_{n}\right)^{T}\right|=\max _{1 \leqslant i \leqslant n}\left|x_{i}\right| .
$$

$C^{p}[0,1]$ is the space of vector-valued functions which are $p$ times continuously differentiable on $[0,1]$ as well as the space of complex-valued matrices, whose elements are $p$ times continuously differentiable. $C^{p}(0,1]$ is defined similarly. For each vector $y \in C^{0}[0,1]$ we define the norm

$$
\|y\|=\max _{0 \leqslant t \leqslant 1}|y(t)|
$$

and for each matrix $A \in C^{0}[0,1],\|A\|$ is the induced norm. We use $C=C[0,1]=$ $C^{0}[0,1]$ and $C(0,1]=C^{0}(0,1]$. For each $y \in C$ the modulus of continuity is defined as

$$
\omega(y ; \delta)=\max _{0 \leqslant t \leqslant 1-\delta}|y(t+\delta)-y(t)|
$$

Let

$$
\pi=\left\{t_{i}, i=0(1) N \mid t_{i}=i \cdot h, t_{N}=1\right\}
$$

be an equidistant partition of $[0,1]$, where $i=0,1, \ldots, N$ is shortly denoted by $i=0(1) N$ and

$$
\Delta=\Delta\left(i_{0}\right)=\left\{t_{i}, i=i_{0}(1) N \mid t_{i}=i \cdot h, t_{N}=1\right\} .
$$

With each partition $\Delta\left(i_{0}\right)$, we associate the linear space $X_{\Delta}$, whose elements $x_{\Delta}$ have the form

$$
x_{\Delta}=\left(x_{i_{0}}, x_{i_{0}+1}, \ldots, x_{N}\right), \quad i_{0} \geqslant 0 \text {, }
$$

where for each $X_{\Delta}, i_{0}$ is a fixed number and $x_{k}=\left(x_{k 1}, x_{k 2}, \ldots, x_{k n}\right)^{T} \in X^{n}$, $k=i_{0}(1) N$. The norm on $X_{\Delta}$ is defined by

$$
\left\|x_{\Delta}\right\|=\max _{i_{0} \leqslant m \leqslant N}\left|x_{m}\right| \text {. }
$$

Finally, we denote by $R_{\Delta}: C \rightarrow X_{\Delta}$ the bounded linear map such that

$$
R_{\Delta} y=\left(y\left(t_{i_{0}}\right), y\left(t_{i_{0}+1}\right), \ldots, y\left(t_{N}\right)\right) .
$$

We now prove the following results.

LEMMA 2.1. Given a complex number $\lambda=\sigma+i \kappa, \sigma>0$ and

$$
\Omega(\lambda)=\{\mu|| \lambda-\mu \mid \leqslant \sigma / 2\} \text {. }
$$


We define for $\mu \in \Omega(\lambda)$

$$
z_{k j}(\mu)= \begin{cases}1, & k=j, \\ \prod_{l=k}\left(t_{l}-\mu h\right) / t_{l}, & 1 \leqslant k<j, j=2(1) N+1 .\end{cases}
$$

Then there exists an $\eta>0$ such that

$$
\left|z_{k j}(\mu)\right| \leqslant \operatorname{const}\left(t_{k} / t_{j}\right)^{\eta}, \quad k \leqslant j, j=1(1) N,
$$

for all $\mu \in \Omega(\lambda)$.

Proof. Since $t_{l}=h l$ for every $l$, we have

$$
\left(t_{l}-\mu h\right) / t_{l}=(l-\mu) / l \text {. }
$$

Let $\mu \in \Omega(\lambda)$ and $l \geqslant l_{0}=\frac{3}{2} \sigma$. Then $|l-\mu| \leqslant|l-\nu|$, where $\nu=\sigma / 2-i(|\kappa|+\sigma / 2)$.

Let $k \geqslant l_{0}$. Then $\left|z_{k j}(\mu)\right| \leqslant\left|v_{k j}\right|$, where

$$
v_{k j}=\prod_{l=k}^{j-1}(l-\nu) / l \text {. }
$$

Using

$$
\Gamma(z+n)=\left(\prod_{k=0}^{n-1}(z+k)\right) \Gamma(z)
$$

and the asymptotic expansion

$$
\Gamma(s+a) / \Gamma(s+b)=s^{a-b}(1+O(1 / s)), \quad \operatorname{Re}(s)>0,
$$

cf. [9], we can rewrite (2.2) and obtain

$$
v_{k j}=\prod_{l=k}^{j-1}(l-\nu) / l=\Gamma(j-\nu) \Gamma(k) / \Gamma(k-\nu) \Gamma(j)=(k / j)^{\nu}(1+O(1 / j)),
$$

which completes the proof for $k \geqslant l_{0}$. The result for $k<l_{0}$ follows now, since $z_{k j}(\mu)=z_{k, l_{0}}(\mu) z_{l_{0}, j}(\mu)$ and $z_{k, l_{0}}$ consists of at most $l_{0}$ terms.

LEMMA 2.2. For every $k>j \geqslant 1$ and $\gamma \in \mathbf{R}$,

$$
\sum_{l=j}^{k-1} h t_{l}^{\gamma-1} \leqslant \begin{cases}\text { const }\left|t_{k}^{\gamma}-t_{j}^{\gamma}\right|, & \gamma \neq 0 \\ \text { const } \ln \left(t_{k} / t_{j}\right), & \gamma=0\end{cases}
$$

Proof. The result follows from the inequality

$$
h t_{l}^{\gamma-1} \leqslant \text { const } \int_{t_{l}}^{t_{t^{+}}} s^{\gamma-1} d s
$$

We also use the fact that, for a matrix $A$ and an analytic function $\zeta(\lambda)$, the matrix function $\zeta(A)$ can be represented as

$$
\zeta(A)=\frac{1}{2 \pi i} \int_{\Gamma} \zeta(\lambda)(\lambda I-A)^{-1} d \lambda,
$$

where $\Gamma$ is a closed curve which contains all eigenvalues of $A$. 
3. Analytical Results. In this section we recapitulate the analytical properties of the solutions of linear problems.

Consider the linear boundary value problem

$$
\begin{gathered}
y^{\prime \prime}(t)-\frac{A_{1}(t)}{t} y^{\prime}(t)-\frac{A_{0}(t)}{t^{2}} y(t)=f(t), \quad 0<t \leqslant 1, \\
B_{0} Y(0)+B_{1} Y(1)=\beta . \quad Y(t)=\left(y(t), y^{\prime}(t)\right)^{T} .
\end{gathered}
$$

By the linear transformation $z=\left(y(t), t y^{\prime}(t)\right)^{T}$, applied to (3.1), we obtain the following boundary value problem of the first order

$$
\begin{gathered}
z^{\prime}(t)=\frac{1}{t}\left[\begin{array}{cc}
0 & I \\
A_{0}(t) & I+A_{1}(t)
\end{array}\right] z(t)+t\left[\begin{array}{c}
0 \\
f(t)
\end{array}\right] \equiv \frac{1}{t} M(t) z(t)+t f(t) \\
B_{0} Y(0)+B_{1} Y(1)=\beta
\end{gathered}
$$

We now consider the case, when $f \in C$ and $A_{0}(t), A_{1}(t)$ can be written as

$$
A_{0}(t)=A_{0}+t^{\nu} C_{0}(t), \quad A_{1}(t)=A_{1}+t^{\nu} C_{1}(t), \quad \nu \geqslant 1,
$$

where $A_{0}, A_{1}$ are constant $n \times n$ matrices and $C_{0}, C_{1} \in C$. By (3.3) the system (3.2) is equivalent to

$$
\begin{gathered}
z^{\prime}(t)=\frac{1}{t}\left[\begin{array}{cc}
0 & I \\
A_{0} & I+A_{1}
\end{array}\right] z(t)+t^{\nu-1}\left[\begin{array}{cc}
0 & 0 \\
C_{0}(t) & C_{1}(t)
\end{array}\right] z(t)+t\left[\begin{array}{c}
0 \\
f(t)
\end{array}\right] \\
\equiv \frac{1}{t} M z(t)+t^{\nu-1} \stackrel{\circ}{C}(t) z(t)+t \stackrel{\circ}{f}(t), \quad 0<t \leqslant 1 \\
B_{0} Y(0)+B_{1} Y(1)=\beta .
\end{gathered}
$$

We denote by $R$ the spectral projection onto the eigenspace of $M=M(0)$, corresponding to an eigenvalue $\lambda=0$, and by $S$ the spectral projection onto the invariant subspace of $M$ associated with the eigenvalues with positive real parts. We set

$$
P=R+S, \quad Q=I-P \text {. }
$$

Finally, we define

$$
S=U+V+T
$$

where $U$ is the spectral projection onto the eigenspace of $M$ corresponding to the eigenvalue $\lambda=1$, and $V$ is the spectral projection onto the invariant subspace associated with eigenvalues, whose real parts are greater than one.

Let $E$ be the $2 n \times 2 n$ matrix of (generalized) eigenvectors of $M$ such that $M=E J E^{-1}$.

Additionally, for any $2 n \times 2 n$ matrix $G$ we denote by $G_{1}$ the $n \times 2 n$ matrix consisting of the $n$ first rows of $G$ and by $G_{2}$ the $n \times 2 n$ matrix consisting of the $n$ last rows of $G$.

We make the following asumptions:

$$
\text { L.3.1. } Q z(0)=0 \text {. }
$$

L.3.2. $B_{01} T_{1}^{E}=0$,

where $T_{1}^{E}$ is the $n \times i$ matrix consisting of nonzero columns of $T_{1} E, i=\operatorname{rank}[T]$ and $B_{01}$ is the $m \times n$ matrix consisting of the last columns of $B_{0}$. 
L.3.3. Let us denote by $\tilde{P}$ the $2 n \times m$ matrix consisting of the linearly independent columns of $P$, where $m=\operatorname{rank}[P]$. Then the $m \times m$ matrix

$$
B_{0}\left[\begin{array}{c}
R_{1} \\
U_{1} M
\end{array}\right] \tilde{P}+B_{1} \tilde{P}
$$

has to be nonsingular.

THEOREM 3.1. If L.3.1, L.3.2 and L.3.3 hold, then (3.1) has a unique continuous solution

$$
y(t)=I_{1} z(t) \text { and } y \in C \cap C^{2}(0,1] .
$$

We note that L.3.1 is the necessary condition for $z$ to be continuous. We have to assume L.3.2 since

$$
\lim _{t \rightarrow 0} y_{T}^{\prime}(t)=\lim _{t \rightarrow 0} T_{1} z^{\prime}(t)=\infty
$$

and L.3.3 is necessary for $y$ to be unique.

Finally, we formulate the smoothness properties of $y$. Let $f, C_{1} \in C^{p}$ and $C_{0} \in C^{p+1}$. Then

(3.5) if the real parts of all eigenvalues of $M$ are nonpositive, $y \in C^{p+2}[0,1]$,

$$
\begin{array}{rlrl}
\text { if } p<\sigma_{+} \leqslant p+1, & y & \in C^{p} \cap C^{p+1}(0,1], \\
\text { if } p+1<\sigma_{+} \leqslant p+2, & y & \in C^{p+1} \cap C^{p+2}(0,1], \\
& \text { if } \sigma_{+}>p+2, & y & \in C^{p+2},
\end{array}
$$

where $\sigma_{+}$is the smallest of the positive real parts of the eigenvalues of $M$.

Clearly, all results are valid if $(3.1)$ is a boundary value problem with constant coefficient matrices $A_{0}(t) \equiv A_{0}$ and $A_{1}(t) \equiv A_{1}$.

4. Numerical Results. Consider a partition $\pi$ as defined in Section 2. Then the standard three-point discretization for (3.1) is

$$
\begin{gathered}
\text { (4.1a) } L_{i} y_{\Delta}:=\frac{y_{i+1}-2 y_{i}+y_{i-1}}{h^{2}}-\frac{A_{1}\left(t_{i}\right)}{t_{i}}\left(\frac{y_{i+1}-y_{i-1}}{2 h}\right)-\frac{A_{0}\left(t_{i}\right)}{t_{i}^{2}} y_{i}=f\left(t_{i}\right), \\
\quad i=1(1) N-1 . \\
B_{0} Y_{0}+B_{1} Y_{N}=\beta,
\end{gathered}
$$

where $Y_{0}=\left(y_{0}, y_{0}^{\prime}\right)^{T}, Y_{N}=\left(y_{N}, y_{N}^{\prime}\right)^{T}$, and where by $y_{0}^{\prime}, y_{N}^{\prime}$ we denote approximations for $y^{\prime}(0)$ and $y^{\prime}(1)$, respectively. Without loss of generality, we assume that the boundary conditions, which are necessary for the solution of (3.1) to be continuous (and equivalent to $Q z(0)=0$ ), are given by

$$
\tilde{Q} Y_{0}=0 \text {, }
$$

where $\tilde{Q}$ is a constant $r \times 2 n$ matrix and $r=\operatorname{rank}[Q]$. For a wide class of problems appearing in practice, we typically have either $y(0)=0$ or $y^{\prime}(0)=0$; see [7], [14]. To approximate $y^{\prime}(1)$ we choose $y_{N}^{\prime}=\left(y_{N+1}-y_{N-1}\right) / 2 h$ and complete the difference scheme (4.1a) by one more equation for $i=N$. The choice of $y_{0}^{\prime}$ is not that simple, because we cannot include the point $t_{-1}=-h$ into the scheme. However, we can remedy the situation if the solution $y$ is smooth enough, which is valid for many practical problems. 
Now we study the case when the coefficient matrices $A_{0}(t)$ and $A_{1}(t)$ are constant. Let $y \in C^{2}$. Then Taylor's theorem applied to Eq. (3.1a) yields

$$
\lim _{t \rightarrow 0}\left(y^{\prime \prime}(t)-\frac{A_{1}}{t}\left(y^{\prime}(0)+t y^{\prime \prime}(\eta)\right)-\frac{A_{0}}{t^{2}}\left(y(0)+t y^{\prime}(0)+\frac{t^{2}}{2} y^{\prime \prime}(\rho)\right)\right)=f(0),
$$

where $0<\eta, \rho<t$, and therefore

$$
A y^{\prime \prime}(0):=\left(I-A_{1}-A_{0} / 2\right) y^{\prime \prime}(0)=f(0)
$$

if and only if

$$
\left(A_{1}+A_{0}\right) y^{\prime}(0)=0, \quad A_{0} y(0)=0 .
$$

Provided that the last conditions hold and $A^{-1}$ exists, the natural approximation for $y^{\prime}(0)$ is

$$
y_{0}^{\prime}=\left(y_{1}-y_{0}\right) / h-h A^{-1} f(0) / 2 .
$$

If (4.2a) is not well defined, another approximation must be taken; for instance, by the polynomial interpolation we obtain immediately

$$
y_{0}^{\prime}=\left(-y_{2}+4 y_{1}-3 y_{0}\right) / 2 h .
$$

Both (4.2a) and (4.2b) are $O\left(h^{2}\right)$ approximations for $y^{\prime}(0)$, if $y \in C^{3}$.

We saw in Section 3 that the fundamental properties of (3.1) have been deduced via an investigation of the associated first order system (3.2). This is also the case for the numerical scheme, and therefore we introduce the transformation

$$
u_{1, i-1}=y_{i-1}, \quad u_{2, i-1}=i\left(y_{i}-y_{i-1}\right), \quad i=1(1) N .
$$

From (4.3) we have immediately

$$
\begin{aligned}
u_{1, i} & =u_{1, i-1}+\frac{1}{i} u_{2, i-1}, & i & =1(1) N, \\
y_{i} & =\frac{u_{2, i-1}}{i}+u_{1, i-1}, & i & =1(1) N .
\end{aligned}
$$

We now rewrite (4.1a) as follows

$$
\begin{aligned}
\left(I-\frac{A_{1}}{2 i}\right) y_{i+1}-\left(2 I+\frac{A_{0}}{i^{2}}\right) y_{i}+\left(I+\frac{A_{1}}{2 i}\right) y_{i-1}=h^{2} f\left(t_{i}\right), & \\
& i=1(1) N-1,
\end{aligned}
$$

and substitute (4.3) and (4.5) into (4.6) obtaining

$$
\begin{aligned}
\left(I-\frac{A_{1}}{2 i}\right)\left(\frac{u_{2, i}}{i+1}+u_{1, i}\right) & -\left(2 I+\frac{A_{0}}{i^{2}}\right)\left(\frac{u_{2, i-1}}{i}+u_{1, i-1}\right) \\
& +\left(I+\frac{A_{1}}{2 i}\right) u_{1, i-1}=h^{2} f\left(t_{i}\right), \quad i=1(1) N .
\end{aligned}
$$

We now make the following assumption.

A.4.1. The matrices

$$
\left(I-\frac{A_{1}}{2 i}\right)^{-1}, \quad 1 \leqslant i \leqslant \frac{\left\|A_{1}\right\|}{2},
$$

exist. We note that $\left\|A_{1} / 2 i\right\|<1$ for each $\left\|A_{1}\right\| / 2<i \leqslant N$, and thus the matrices $I-A_{1} / 2 i$ are regular. 
We now rewrite (4.7) and have

$$
\begin{aligned}
\left(I-\frac{A_{1}}{2 i}\right) \frac{u_{2, i}}{i+1}= & -\left(I-\frac{A_{1}}{2 i}\right) u_{1, i}+\left(2 I+\frac{A_{0}}{i^{2}}\right)\left(\frac{u_{2, i-1}}{i}+u_{1, i-1}\right) \\
& -\left(I+\frac{A_{1}}{2 i}\right) u_{1, i-1}+h^{2} f\left(t_{i}\right), \quad i=1(1) N .
\end{aligned}
$$

This implies

$$
\begin{aligned}
u_{2, i}= & -(i+1)\left(\frac{u_{2, i-1}}{i}+u_{1, i-1}\right) \\
& +(i+1)\left(I-\frac{A_{1}}{2 i}\right)^{-1}\left(2 I+\frac{A_{0}}{i^{2}}\right)\left(\frac{u_{2, i-1}}{i}+u_{1, i-1}\right) \\
& -(i+1)\left(I-\frac{A_{1}}{2 i}\right)^{-1}\left(I+\frac{A_{1}}{2 i}\right) u_{1, i-1}+(i+1)\left(I-\frac{A_{1}}{2 i}\right)^{-1} h^{2} f\left(t_{i}\right) \\
= & \frac{i+1}{i}\left[-I+\left(I-\frac{A_{1}}{2 i}\right)^{-1}\left(2 I+\frac{A_{0}}{i^{2}}\right)\right] u_{2, i-1} \\
& +(i+1)\left[-I+\left(I-\frac{A_{1}}{2 i}\right)^{-1}\left(\left(2 I+\frac{A_{0}}{i^{2}}\right)-\left(I+\frac{A_{1}}{2 i}\right)\right)\right] u_{1, i-1} \\
& +\left(I-\frac{A_{1}}{2 i}\right)^{-1} h t_{i+1} f\left(t_{i}\right) \\
\equiv & \theta_{1} u_{1, i-1}+\theta_{2} u_{2, i-1}+\left(I-\frac{A_{1}}{2 i}\right)^{-1} h t_{i+1} f\left(t_{i}\right) .
\end{aligned}
$$

For $\theta_{1}$ and $\theta_{2}$ we have

$$
\begin{aligned}
\theta_{1}= & (i+1)\left(I-\frac{A_{1}}{2 i}\right)^{-1} \frac{A_{0}}{i^{2}}=\frac{A_{0}}{i}+\frac{1}{i^{2}}\left(I-\frac{A_{1}}{2 i}\right)^{-1}\left(\frac{A_{1}}{2}+I\right) A_{0}, \\
\theta_{2}= & \left(1+\frac{1}{i}\right)\left(I-\frac{A_{1}}{2 i}\right)^{-1}\left(I+\frac{A_{1}}{2 i}+\frac{A_{0}}{i^{2}}\right) \\
= & I+\frac{1}{i}\left(I+A_{1}\right)+\left(I-\frac{A_{1}}{2 i}\right)^{-1}\left(\frac{A_{1}}{i}+\frac{A_{0}}{i^{2}}\right) \\
& +\frac{1}{i}\left[\left(I-\frac{A_{1}}{2 i}\right)^{-1}\left(\frac{A_{1}}{i}+\frac{A_{0}}{i^{2}}\right)-A_{1}\right] \\
= & I+\frac{1}{i}\left(I+A_{1}\right)+\frac{1}{i^{2}}\left(I-\frac{A_{1}}{2 i}\right)^{-1}\left(\left(1+\frac{1}{i}\right) A_{0}+\left(I+\frac{A_{1}}{2}\right) A_{1}\right),
\end{aligned}
$$

and finally

$$
\begin{aligned}
u_{2, i}= & \left(\frac{A_{0}}{i}+\frac{1}{i^{2}} \Theta_{1}(i)\right) u_{1, i-1}+\left(I+\frac{1}{i}\left(I+A_{1}\right)+\frac{1}{i^{2}} \Theta_{2}(i)\right) u_{2, i-1} \\
& +\Theta_{3}(i) t_{i+1} h f\left(t_{i}\right), \quad i=1(1) N
\end{aligned}
$$

where

$$
\Theta_{1}(i)=\left(I-\frac{A_{1}}{2 i}\right)^{-1}\left(I+\frac{A_{1}}{2}\right) A_{0}
$$




$$
\begin{aligned}
& \Theta_{2}(i)=\left(I-\frac{A_{1}}{2 i}\right)^{-1}\left[\left(1+\frac{1}{i}\right) A_{0}+\left(I+\frac{A_{1}}{2}\right) A_{1}\right], \\
& \Theta_{3}(i)=\left(I-\frac{A_{1}}{2 i}\right)^{-1} .
\end{aligned}
$$

(4.4) and (4.9) yield the following system for

$$
\begin{aligned}
& u_{i}=\left(u_{1, i}, u_{2, i}\right)^{T}, \quad i=1(1) N, \\
& u_{i}=u_{i-1}+\frac{1}{i} M u_{i-1}+\frac{1}{i^{2}} \Theta_{4}(i) u_{i-1}+t_{i+1} h \Theta_{5}(i) \stackrel{\circ}{f}\left(t_{i}\right),
\end{aligned}
$$

where

$$
\Theta_{4}(i)=\left[\begin{array}{cc}
0 & 0 \\
\Theta_{1}(i) & \Theta_{2}(i)
\end{array}\right], \quad \Theta_{5}(i)=\left[\begin{array}{cc}
0 & 0 \\
0 & \Theta_{3}(i)
\end{array}\right] .
$$

Due to $J=E^{-1} M E$, we have for the vector $v_{i}=E^{-1} u_{i}$ the following equation associated with (4.11)

$$
v_{i}=v_{i-1}+\frac{1}{i} J v_{i-1}+\frac{1}{i^{2}} \Theta(i) v_{i-1}+t_{i+1} h \Psi(i) \stackrel{\circ}{f}\left(t_{i}\right),
$$

where

$$
\Theta(i)=E^{-1} \Theta_{4}(i) E, \quad \Psi(i)=E^{-1} \Theta_{5}(i), \quad i=1(1) N .
$$

As a first step in the analysis of (4.12) we study the growth of solutions of the system of difference equations

$$
v_{i}=v_{i-1}+\frac{1}{i} J v_{i-1}+h^{\alpha+1} t_{i}^{\gamma+1} r_{i}, \quad i=1(1) N,
$$

where $\alpha, \gamma$ are real numbers, $r_{i}$ is a $2 n$-vector and $J$ is a $2 n \times 2 n$ Jordan box

$$
J=\left[\begin{array}{lll}
\lambda & 1 & \\
& & 1
\end{array}\right], \quad \lambda=\sigma+i \kappa .
$$

We consider the three cases $\sigma<0, \lambda=0, \sigma>0$ separately.

Case $1 . \sigma<0$. Let $v_{i_{0}}=\delta, 0 \leqslant i_{0} \leqslant i$. Then

$$
\begin{aligned}
& v_{i}= \prod_{l=i}^{i_{0}+1}\left(I+\frac{1}{l} J\right) \delta+\sum_{k=i_{0}+1}^{i-1}\left(\prod_{l=i}^{k+1}\left(I+\frac{1}{l} J\right)\right) h^{\alpha+1} t_{k}^{\gamma+1} r_{k} \\
&+h^{\alpha+1} t_{i}^{\gamma+1} r_{i} \\
& \equiv Z_{i, i_{0}+1} \delta+\sum_{k=i_{0}+1}^{i-1} Z_{i, k+1} h^{\alpha+1} t_{k}^{\gamma+1} r_{k}+h^{\alpha+1} t_{i}^{\gamma+1} r_{i}, \\
& i=\left(i_{0}+1\right)(1) N,
\end{aligned}
$$

where

$$
Z_{j, k+1}=z_{k+1, j+1}(-J)=\frac{1}{2 \pi i} \int_{\Gamma} z_{k+1, j+1}(-\lambda)(\lambda I-J)^{-1} d \lambda
$$

and $\Gamma=\{\mu|| \lambda-\mu \mid=-\sigma / 2\}$. From Lemma 2.1 we have

$$
\left|Z_{i, k+1}\right| \leqslant \operatorname{const}\left(t_{k+1} / t_{i+1}\right)^{\eta}, \quad \eta>0
$$


and

(4.15) $\left|v_{i}\right| \leqslant \operatorname{const}\left\{|\delta|+\sum_{k=i_{0}+1}^{i}\left(t_{k+1} / t_{i+1}\right)^{\eta} h^{\alpha+1} t_{k}^{\gamma+1}\left|r_{k}\right|\right\}, \quad i=\left(i_{0}+1\right)(1) N$

Furthermore

$$
\begin{aligned}
\sum_{k=i_{0}+1}^{i}\left(t_{k+1} / t_{i+1}\right)^{\eta} h^{\alpha+1} t_{k}^{\gamma+1}\left|r_{k}\right| & \\
& \leqslant \begin{cases}\operatorname{const}\left(t_{i+1}\right)^{-\eta} h^{\alpha} \sum_{k=i_{0}+1}^{i} h t_{k}^{\gamma+\eta+1}\left\|r_{\Delta}\right\|, & \gamma \geqslant-2 \\
h^{\alpha} \sum_{k=i_{0}+1}^{i} h t_{k}^{\gamma+1}\left\|r_{\Delta}\right\|, & \gamma<-2\end{cases}
\end{aligned}
$$

and by Lemma 2.2

$$
\leqslant \begin{cases}\operatorname{const}\left(t_{i+1}\right)^{-\eta} h^{\alpha}\left|t_{i+1}^{\gamma+\eta+2}-t_{i_{0}+1}^{\gamma+\eta+2}\right|\left\|r_{\Delta}\right\|, & \gamma>-2, \\ \operatorname{const}\left(t_{i+1}\right)^{-\eta} h^{\alpha}\left|t_{i+1}^{\eta}-t_{i_{0}+1}^{\eta}\right|\left\|r_{\Delta}\right\|, & \gamma=-2, \\ \operatorname{const} h^{\alpha}\left|t_{i+1}^{\gamma+2}-t_{i_{0}+1}^{\gamma+2}\right|\left\|r_{\Delta}\right\|, & \gamma<-2 .\end{cases}
$$

Finally, we have

$$
\left|v_{i}\right| \leqslant \begin{cases}\operatorname{const}\left\{|\delta|+h^{\alpha} t_{i}^{\gamma+2}\left\|r_{\Delta}\right\|\right\}, & \gamma>-2 \\ \operatorname{const}\left\{|\delta|+h^{\alpha}\left\|r_{\Delta}\right\|\right\}, & \gamma=-2 \\ \operatorname{const}\left\{|\delta|+h^{\alpha} t_{i_{0}}^{\gamma+2}\left\|r_{\Delta}\right\|\right\}, & \gamma<-2\end{cases}
$$

Case 2. $\lambda=0$. Let $v_{N, 1}=\delta_{1}$ and $v_{i_{0}, k}=\delta_{k}, k=2(1) 2 n$. We consider the system (4.13) in its component form, and for the last component $v_{i, 2 n}$ we obtain

$$
v_{i, 2 n}=v_{i_{0}, 2 n}+\sum_{k=i_{0}+1}^{i} h^{\alpha+1} t_{k}^{\gamma+1} r_{k, 2 n}, \quad 0 \leqslant i_{0}<i \leqslant N
$$

and by Lemma 2.2

$$
\left|v_{i, 2 n}\right| \leqslant \begin{cases}\left|\delta_{2 n}\right|+\text { const } h^{\alpha} t_{i}^{\gamma+2}\left\|r_{\Delta}\right\|, & \gamma>-2 \\ \left|\delta_{2 n}\right|+\text { const } h^{\alpha}\left|\ln t_{i_{0}}\right|\left\|r_{\Delta}\right\|, & \gamma=-2 \\ \left|\delta_{2 n}\right|+\text { const } h^{\alpha} t_{i_{0}}^{\gamma+2}\left\|r_{\Delta}\right\|, & \gamma<-2\end{cases}
$$

For the $(2 n-1)$ th component we have

$$
v_{i, 2 n-1}=v_{i_{0}, 2 n-1}+\sum_{k=i_{0}+1}^{i} \frac{1}{k} v_{k-1,2 n}+\sum_{k=i_{0}+1}^{i} h^{\alpha+1} t_{k}^{\gamma+1} r_{k, 2 n-1}
$$

and there is by (4.18) and Lemma 2.2

$$
\left|v_{i, 2 n-1}\right| \leqslant \begin{cases}\operatorname{const}\left\{\left|\delta_{2 n-1}\right|+\left|\delta_{2 n}\right|\left|\ln t_{i_{0}}\right|+h^{\alpha} t_{i}^{\gamma+2}\left\|r_{\Delta}\right\|\right\}, & \gamma>-2, \\ \operatorname{const}\left\{\left|\delta_{2 n-1}\right|+\left|\delta_{2 n}\right|\left|\ln t_{i_{0}}\right|+h^{\alpha}\left(\left|\ln t_{i_{0}}\right|+\left|\ln t_{i_{0}}\right|^{2}\right)\left\|r_{\Delta}\right\|\right\}, & \gamma=-2, \\ \operatorname{const}\left\{\left|\delta_{2 n-1}\right|+\left|\delta_{2 n}\right|\left|\ln t_{i_{0}}\right|+h^{\alpha} t_{i_{0}}^{\gamma+2}\left(1+\left|\ln t_{i_{0}}\right|\right)\left\|r_{\Delta}\right\|\right\}, & \gamma<-2 .\end{cases}
$$


Clearly, continuing this process we obtain

$$
\left|v_{i, r}\right| \leqslant \begin{cases}\text { const }\left\{\sum_{k=r}^{2 n}\left|\delta_{k}\right|\left|\ln t_{i_{0}}\right|^{k-r}+h^{\alpha} t_{i}^{\gamma+2}\left\|r_{\Delta}\right\|\right\}, & \gamma>-2 \\ \operatorname{const}\left\{\sum_{k=r}^{2 n}\left|\delta_{k}\right|\left|\ln t_{i_{0}}\right|^{k-r}+h^{\alpha} \sum_{k=1}^{2 n-r+1}\left|\ln t_{i_{0}}\right|^{k}\left\|r_{\Delta}\right\|\right\}, & \gamma=-2 \\ \operatorname{const}\left\{\sum_{k=r}^{2 n}\left|\delta_{k}\right|\left|\ln t_{i_{0}}\right|^{k-r}+h^{\alpha} t_{i_{0}}^{\gamma+2} \sum_{k=0}^{2 n-r}\left|\ln t_{i_{0}}\right|^{k}\left\|r_{\Delta}\right\|\right\}, & \gamma<-2\end{cases}
$$

for $r=2(1) 2 n$ and $i=\left(i_{0}+1\right)(1) N$.

Finally, for $r=1$

$$
v_{i, 1}=v_{N, 1}-\sum_{k=i+1}^{N} \frac{1}{k} v_{k-1,2}-\sum_{k=i+1}^{N} h^{\alpha+1} t_{k}^{\gamma+1} r_{k, 1},
$$

and the following estimate is a result of (4.19) for $r=2$ and Lemma 2.2

$(4.20) \quad\left|v_{i, 1}\right| \leqslant\left\{\begin{array}{cl}\operatorname{const}\left\{\left|\delta_{1}\right|+\sum_{k=2}^{2 n}\left|\delta_{k}\right|\left|\ln t_{i_{0}}\right|^{k-1}+h^{\alpha}\left(1-t_{i}^{\gamma+2}\right)\left\|r_{\Delta}\right\|\right\}, & \gamma>-2, \\ \operatorname{const}\left\{\left|\delta_{1}\right|+\sum_{k=2}^{2 n}\left|\delta_{k}\right|\left|\ln t_{i_{0}}\right|^{k-1}+h^{\alpha} \sum_{k=1}^{2 n}\left|\ln t_{i_{0}}\right|^{k}\left\|r_{\Delta}\right\|\right\}, & \gamma=-2, \\ \operatorname{const}\left\{\left|\delta_{1}\right|+\sum_{k=2}^{2 n}\left|\delta_{k}\right|\left|\ln t_{i_{0}}\right|^{k-1}\right. & \\ \left.+h^{\alpha}\left(t_{i}^{\gamma+2}+t_{i_{0}}^{\gamma+2} \sum_{k=1}^{2 n-1}\left|\ln t_{i_{0}}\right|^{k}\right)\left\|r_{\Delta}\right\|\right\}, & \gamma<-2 .\end{array}\right.$

Clearly, if $\left|\delta_{0}\right| \equiv \max _{2 \leqslant k \leqslant 2 n}\left|\delta_{k}\right|$, then

(4.21) $\left\|v_{\Delta}\right\| \leqslant \begin{cases}\operatorname{const}\left\{\left|\delta_{1}\right|+\left|\delta_{0}\right||\ln h|^{2 n-1}+h^{\alpha}\left\|r_{\Delta}\right\|\right\}, & \gamma>-2, \\ \operatorname{const}\left\{\left|\delta_{1}\right|+\left|\delta_{0}\right||\ln h|^{2 n-1}+h^{\alpha}|\ln h|^{2 n}\left\|r_{\Delta}\right\|\right\}, & \gamma=-2, \\ \operatorname{const}\left\{\left|\delta_{1}\right|+\left|\delta_{0}\right||\ln h|^{2 n-1}+h^{\alpha+\gamma+2}|\ln h|^{2 n-1}\left\|r_{\Delta}\right\|\right\}, & \gamma<-2 .\end{cases}$

Case 3. $\sigma>0$. For the system (4.13) and $v_{N}=\delta$ we have

$$
\begin{aligned}
& v_{i}=\left(I+\frac{1}{i+1} J\right)^{-1}\left(v_{i+1}-h^{\alpha+1} t_{i+1}^{\gamma+1} r_{i+1}\right) \\
& =\prod_{k=i+1}^{N}\left(I+\frac{1}{k} J\right)^{-1} \delta-\sum_{k=i+1}^{N}\left(\prod_{l=i+1}^{k}\left(I+\frac{1}{l} J\right)^{-1}\right) h^{\alpha+1} t_{k}^{\gamma+1} r_{k}, \quad i=0(1) N-1 .
\end{aligned}
$$

Since $\sigma>0,(I+J / k)^{-1}$ exist for each $k$ and by the definition from Lemma 2.1 we can rewrite the last expression, obtaining

$$
v_{i}=Z_{i+1, N} \delta-\sum_{k=i+1}^{N} Z_{i+1, k} h^{\alpha+1} t_{k}^{\gamma+1} r_{k}
$$


where

$$
Z_{j+1, k}=z_{j+1, k+1}(J)=\frac{1}{2 \pi i} \int_{\Gamma} z_{j+1, k+1}(\lambda)(\lambda I-J)^{-1} d \lambda
$$

and $\Gamma=\{\mu|| \lambda-\mu \mid=\sigma / 2\}$. It follows from Lemma 2.1 that there exists a constant $0<\eta<1$, such that

$$
\left|v_{i}\right| \leqslant \text { const }\left\{t_{i}^{\eta}|\delta|+\sum_{k=i+1}^{N}\left(t_{i+1} / t_{k+1}\right)^{\eta} h^{\alpha+1} t_{k}^{\gamma+1}\left|r_{k}\right|\right\}, \quad i=0(1) N-1,
$$

and from Lemma 2.2

$$
\left|v_{i}\right| \leqslant \begin{cases}\operatorname{const}\left\{t_{i}^{\eta}|\delta|+h^{\alpha}\left\|r_{\Delta}\right\|\right\}, & \gamma>-2, \\ \operatorname{const}\left\{t_{i}^{\eta}|\delta|+h^{\alpha}\left\|r_{\Delta}\right\|\right\}, & \gamma=-2, \quad i=0(1) N-1 \\ \operatorname{const}\left\{t_{i}^{\eta}|\delta|+h^{\alpha} t_{i}^{\gamma+2}\left\|r_{\Delta}\right\|\right\}, & \gamma<-2 .\end{cases}
$$

Finally, we consider the iteration scheme, which is implied by (4.12) and has the form

$$
\begin{gathered}
v_{i}^{(k+1)}-v_{i-1}^{(k+1)}-\frac{1}{i} J v_{i-1}^{(k+1)}=\frac{1}{i^{2}} \Theta(i) v_{i-1}^{(k)}+t_{i+1} \Psi(i) h \stackrel{\circ}{f}\left(t_{i}\right), \\
\\
Q v_{i_{0}}^{(k+1)}=\delta_{0}, \quad P v_{N}^{(k+1)}=\delta_{N} .
\end{gathered}
$$

Formally, we can write this as

where $G: X_{\Delta} \rightarrow X_{\Delta}$ is a bounded linear map. Additionally, we use the following notation:

$$
G_{i} V^{(k)} \equiv\left(G V^{(k)}\right)_{i} \equiv v_{i}^{(k+1)} .
$$

We can now formulate three lemmas dealing with the cases when all eigenvalues of $M$ have negative real parts, are zero or have positive real parts.

LEMMA 4.1. Let all eigenvalues of $M$ have negative real parts.

(i) For each $t_{i_{0}}=\varepsilon>0$, there exists an $h(\varepsilon)$ such that, for each $h \in(0, h(\varepsilon))$, the system (4.11) has a unique solution for each $v_{i_{0}}=\delta$ and $f_{\Delta}$. Furthermore

$$
\left\|u_{\Delta}\right\| \leqslant \text { const }\left\{|\delta|+\left\|f_{\Delta}\right\|\right\} \text {. }
$$

(ii) Let $y_{\Delta}$ be a solution of (4.1a). Then

$$
\begin{array}{ll}
\text { (a) }\left\|y_{\Delta}-R_{\Delta} y\right\| \leqslant \text { const } \omega(f, h), & \text { if } f \in C, \\
\text { (b) }\left\|y_{\Delta}-R_{\Delta} y\right\| \leqslant \text { const } h^{2}, & \text { if } f \in C^{2} .
\end{array}
$$

All constants appearing in the estimates (i) and (ii) do not depend on $\varepsilon$.

Proof. (i) We consider the system (4.12) and show that $G$ is contracting on $X_{\Delta}$. Let $V$ and $W$ be in $X_{\Delta}$. Then by (4.15)

$$
\left|G_{i} V-G_{i} W\right| \leqslant \mathrm{const} \sum_{r=i_{0}+1}^{i}\left(t_{r+1} / t_{i+1}\right)^{\eta} h^{2} t_{r}^{-2}\|\Theta(r)\|\left|V_{r-1}-W_{r-1}\right|,
$$


and it follows from (4.16) that, for $\alpha=1$ and $\gamma=-3$,

$$
\left|G_{i} V-G_{i} W\right| \leqslant C \frac{h}{t_{i_{0}}}\left\|\Theta_{\Delta}\right\|\left\|(V-W)_{\Delta}\right\|,
$$

where $C=$ const. Clearly, for each $t_{i_{0}}=\varepsilon>0$, there exists an $h(\varepsilon)=\varepsilon / C\left\|\Theta_{\Delta}\right\|$ such that, for each $h<h(\varepsilon)$,

$$
\left\|(G V-G W)_{\Delta}\right\|<\left\|(V-W)_{\Delta}\right\|
$$

and the result follows. The estimate in (i) is obtained now from (4.16) for $\alpha=\gamma=0$ and $u_{i}=E v_{i}$.

(ii)(a) In this case $y \in C^{2}$, if $f \in C$ and $\tilde{Q} Y(0)$ is equivalent to $y(0)=y^{\prime}(0)=0$. Furthermore, it follows from [16, Lemma 3.1] that

$$
\omega\left(y^{\prime \prime}, h\right) \leqslant \text { const } \omega(f, h)
$$

and

$$
\left|y^{\prime}\left(t_{j}\right)-y^{\prime}\left(\eta_{j}\right)\right| \leqslant \text { const } t_{j+1} \omega(f, h), \quad t_{j}<\eta_{j}<t_{j+1} .
$$

Also

$$
\begin{aligned}
L_{j} R_{\Delta} y= & y^{\prime \prime}\left(t_{j}\right)-\frac{A_{1}}{t_{j}} y^{\prime}\left(t_{j}\right)-\frac{A_{0}}{t_{j}^{2}} y\left(t_{j}\right) \\
& +\left(y^{\prime \prime}\left(\eta_{j}\right)-y^{\prime \prime}\left(t_{j}\right)\right)-\frac{A_{1}}{t_{j}}\left(y^{\prime}\left(\xi_{j}\right)-y^{\prime}\left(t_{j}\right)\right),
\end{aligned}
$$

where $t_{j}<\eta_{j}, \xi_{j}<t_{j+1}, j=1(1) N$ and hence

$$
\left|L_{j}\left(R_{\Delta} y-y_{\Delta}\right)\right| \leqslant \text { const } \omega(f, h), \quad j=1(1) N .
$$

Assume (i) to hold for an index $i_{0} \geqslant 0$, and consider the initial conditions

$$
y_{i_{0}}=y_{0}, \quad\left(y_{i_{0}+1}-y_{i_{0}}\right) / h=y^{\prime}(0) \text {. }
$$

Then, it follows by Taylor's theorem applied to (4.27) that

$$
\delta \equiv\left[\begin{array}{c}
y_{i_{0}}-y\left(t_{i_{0}}\right) \\
t_{i_{0}+1}\left[\left(y_{i_{0}+1}-y_{i_{0}}\right) / h-y^{\prime}\left(t_{i_{0}}\right)\right]
\end{array}\right]=O\left(h^{2}\right)
$$

and the result holds by (i).

(ii)(b) Since $y \in C^{4}$ if $f \in C^{2}$, the result follows in a very similar way on noting that

$$
\left|L_{j}\left(R_{\Delta} y-y_{\Delta}\right)\right| \leqslant \operatorname{const}\left\{h^{2} M_{\Delta}^{4}+h^{2} M_{\Delta}^{3} / t_{j}\right\}, \quad j=1(1) N
$$

where $M_{\Delta}^{k}=\max _{1 \leqslant j \leqslant N}\left|y^{(k)}\left(t_{j}\right)\right|, k=3,4$.

LEMMA 4.2. Let all eigenvalues of $M$ be zero.

(i) For each $t_{i_{0}}=\varepsilon>0$, there exists an $h(\varepsilon)$ such that, for each $h \in(0, h(\varepsilon))$, the system (4.11) has a unique solution for each $(I-R) u_{i_{0}}=\delta_{0}, R u_{N}=\delta_{N}$ and $f_{\Delta}$. Furthermore

$$
\left\|u_{\Delta}\right\| \leqslant \operatorname{const}\left\{\left|\delta_{0}\right||\ln h|^{d_{0}-1}+\left|\delta_{N}\right|+\left\|f_{\Delta}\right\|\right\}
$$

where $d_{0}$ is the dimension of the largest Jordan box of $M$. 
(ii) Let $y$ be a solution of (4.1a) subject to the terminal condition $R Y_{N}=R Y(1)$, see [16, Lemma 3.2 and Remark 3.1]. Then

$$
\begin{array}{ll}
\text { (a) }\left\|y_{\Delta}-R_{\Delta} y\right\| \leqslant \mathrm{const} \omega(f, h), & \text { if } f \in C, \\
\text { (b) }\left\|y_{\Delta}-R_{\Delta} y\right\| \leqslant \mathrm{const} h^{2}|\ln h|^{d_{0}-1}, & \text { if } f \in C^{2} .
\end{array}
$$

Proof. (i) Consider the system (4.12) and the iteration scheme defined by (4.24). Then it follows by (4.20) that for $V, W \in X_{\Delta}$ and $\alpha=1, \gamma=-3$,

$$
\left|G_{i} V-G_{i} W\right| \leqslant C \frac{h}{t_{i_{0}}}\left|\ln t_{i_{0}}\right|^{d_{0}-1}\left\|\Theta_{\Delta}\right\|\left\|(V-W)_{\Delta}\right\|,
$$

and the result holds for each $t_{i_{0}}=\varepsilon$ and $h<\varepsilon /\left(C|\ln \varepsilon|^{d_{0}-1}\left\|\Theta_{\Delta}\right\|\right)$. The estimate for $u_{\Delta}$ can be obtained now from (4.20) for $\alpha=\gamma=0$.

(ii) This follows as in Lemma 4.1, since $y \in C^{p+2}$ if $f \in C^{p}, p \geqslant 0$, and $y^{\prime}(t)$ has the same form as in Case 1. Furthermore, $\delta_{N}=R Y_{N}-R Y(1)=0$ and $\delta_{0}=O\left(h^{2}\right)$ yield the result.

LEMMA 4.3. Let all eigenvalues of $M$ have positive real parts.

(i) For each $t_{i_{0}}=\varepsilon>0$, there exists an $h(\varepsilon)$ such that, for each $h \in(0, h(\varepsilon))$, the system (4.11) has a unique solution for each $u_{N}=\delta$ and $f_{\Delta}$. Also

$$
\left\|u_{\Delta}\right\| \leqslant \operatorname{const}\left\{|\delta|+\left\|f_{\Delta}\right\|\right\} \text {. }
$$

(ii) Let $y_{\Delta}$ be a solution of (4.1a) subject to the terminal condition $y_{N}=Y(1)$; see [16, Lemma 3.3].

(a) If $f \in C$, then

$$
\left\|y_{\Delta}-R_{\Delta} y\right\| \leqslant \begin{cases}\operatorname{const}\left\{h^{\sigma_{+}}|\ln h|^{d_{+}-1}+\omega(f, h)\right\}, & 0<\sigma_{+} \leqslant 1, \\ \operatorname{const}\left\{h|\ln h|^{d_{+}-1}+\omega(f, h)\right\}, & 1<\sigma_{+}<2, \\ \operatorname{const}\left\{h|\ln h|^{d_{+}}+\omega(f, h)\right\}, & \sigma_{+}=2, \\ \operatorname{const}\{h+\omega(f, h)\}, & \sigma_{+}>2 .\end{cases}
$$

(b) If $f \in C^{2}$, then

$$
\left\|y_{\Delta}-R_{\Delta} y\right\| \leqslant \begin{cases}\text { const } h^{\sigma_{+}}|\ln h|^{d_{+}-1}, & 0<\sigma_{+}<2, \\ \text { const } h^{2}|\ln h|^{d_{+}}, & \sigma_{+}=2, \\ \text { const } h^{2}, & \sigma_{+}>2 .\end{cases}
$$

Proof. (i) Consider the iteration (4.24). Let $V, W \in X_{\Delta}$. Then it follows from (4.23) for $\alpha=1, \gamma=-3$ that

$$
\left|G_{i} V-G_{i} W\right| \leqslant C \frac{h}{t_{i}}\left\|\Theta_{\Delta}\right\|\left\|(V-W)_{\Delta}\right\|, \quad i_{0} \leqslant i \leqslant N
$$

and $G$ is contracting on $X_{\Delta}$ for each $t_{i_{0}}=\varepsilon>0$ if $h<\varepsilon / C\left\|\Theta_{\Delta}\right\|$. The estimate follows now from (4.23) for $\alpha=\gamma=0$.

(ii)(a) Consider the system (3.2). Let $\varphi(t)=E^{-1} z(t)$. Then $\varphi$ is a solution of the following problem, $\left(\stackrel{\circ}{g}(t)=E^{-1} f(t)\right)$

$$
\varphi^{\prime}(t)=\frac{1}{t} J \varphi(t)+\operatorname{tg}(t), \quad g \in C .
$$


Let us assume that $J$ consists of one Jordon box of dimension $2 n$, whose eigenvalues are $\lambda=\sigma+i \kappa, \sigma>0$, and investigate $y^{\prime}\left(\eta_{j}\right)-y^{\prime}\left(t_{j}\right)$ and $y^{\prime \prime}\left(\xi_{j}\right)-y^{\prime \prime}\left(t_{j}\right)$. From the mean value theorem, we obtain

$$
\left(\varphi_{r}\left(t_{j+1}\right)-\varphi_{r}\left(t_{j}\right)\right) / h-\varphi_{r}^{\prime}\left(t_{j}\right)=\varphi_{r}^{\prime}\left(\eta_{j r}\right)-\varphi_{r}^{\prime}\left(t_{j}\right),
$$

where $\eta_{j r}=t_{j}+\varepsilon_{j r} h,\left|\varepsilon_{j r}\right|<1, r=1(1) 2 n$ and $j=0(1) N-1$.

Using (4.31), we have for $\nu_{r}=1$ if $r=1(1) 2 n-1$ and $\nu_{2 n}=0$

$$
\begin{aligned}
\varphi_{r}^{\prime}\left(\eta_{j r}\right)-\varphi_{r}^{\prime}\left(t_{j}\right)= & \lambda\left(\frac{1}{\eta_{j r}} \varphi_{r}\left(\eta_{j r}\right)-\frac{1}{t_{j}} \varphi_{r}\left(t_{j}\right)\right) \\
& +\frac{\nu_{r}}{\eta_{j r}} \varphi_{r+1}\left(\eta_{j r}\right)-\frac{\nu_{r}}{t_{j}} \varphi_{r+1}\left(t_{j}\right)+\eta_{j r} \dot{g}_{r}\left(\eta_{j r}\right)-t_{j} \dot{g}_{r}\left(t_{j}\right) \\
= & \left(\frac{1}{\eta_{j r}}-\frac{1}{t_{j}}\right)\left(\lambda \varphi_{r}\left(\eta_{j r}\right)+\nu_{r} \varphi_{r+1}\left(\eta_{j r}\right)\right) \\
& +\frac{1}{t_{j}}\left[\lambda\left(\varphi_{r}\left(\eta_{j r}\right)-\varphi_{r}\left(t_{j}\right)\right)+\nu_{r}\left(\varphi_{r+1}\left(\eta_{j r}\right)-\varphi_{r+1}\left(t_{j}\right)\right)\right] \\
& +\eta_{j r} \stackrel{\circ}{r}_{r}\left(\eta_{j r}\right)-t_{j} \dot{g}_{r}\left(t_{j}\right) \\
= & O\left(\left|\varphi\left(t_{j}\right)\right| / h j^{2}\right)+O(\omega(\varphi, h) / h j)+O\left(t_{j} \omega(\stackrel{\circ}{g}, h)\right) .
\end{aligned}
$$

Clearly,

$$
y^{\prime}\left(\eta_{j}\right)-y^{\prime}\left(t_{j}\right)=O\left(\left|\varphi\left(t_{j}\right)\right| / j t_{j}\right)+O\left(\omega(\varphi, h) / t_{j}\right)+O\left(t_{j} \omega(f, h)\right)
$$

and

$$
y^{\prime \prime}\left(\xi_{j}\right)-y^{\prime \prime}\left(t_{j}\right)=O\left(\left|\varphi\left(t_{j}\right)\right| / j t_{j}^{2}\right)+O\left(\omega(\varphi, h) / t_{j}^{2}\right)+O(\omega(f, h)) .
$$

Thus

$$
\begin{aligned}
\left|L_{j}\left(y_{\Delta}-R_{\Delta} y\right)\right| \leqslant \operatorname{const}\left\{h\left|\varphi\left(t_{j}\right)\right| / t_{j}^{3}+\omega(\varphi, h) / t_{j}^{2}+\omega(f, h)\right\}, & \\
& j=1(1) N-1,
\end{aligned}
$$

and $y_{N}^{\prime}-y^{\prime}(1)=O(h), y_{N}-y(1)=0$.

Let $0<\sigma<2$. Hence, we have from [16, Lemma 3.3]

$$
\begin{aligned}
& \left|\varphi\left(t_{j}\right)\right| \leqslant \text { const } t_{j}^{o}|\ln h|^{2 n-1}, \quad j=1(1) N, \\
& \omega(\varphi, h) \leqslant \begin{cases}\text { const } h^{\sigma}|\ln h|^{2 n-1}, & \sigma \leqslant 1, \\
\text { const } h|\ln h|^{2 n-1}, & 1<\sigma<2 .\end{cases}
\end{aligned}
$$

Let $0<\sigma \leqslant 1$. Then (4.32) is

$$
\left|L_{j}\left(y_{\Delta}-R_{\Delta} y\right)\right| \leqslant \text { const }\left\{h t_{j}^{\sigma-3}|\ln h|^{2 n-1}+h^{\sigma} t_{j}^{-2}|\ln h|^{2 n-1}+\omega(f, h)\right\},
$$

and the first estimate in (ii)(a) follows by (4.23) for $\alpha=1, \gamma=\sigma-3 \leqslant-2 ; \alpha=\sigma$, $\gamma=-2$ and $\alpha=\gamma=0$, respectively. Let $1<\sigma<2$. Then we can write (4.32) as follows

$$
\left|L_{j}\left(y_{\Delta}-R_{\Delta} y\right)\right| \leqslant \operatorname{const}\left\{h t_{j}^{\sigma-3}|\ln h|^{2 n-1}+h t_{j}^{-2}|\ln h|^{2 n-1}+\omega(f, h)\right\},
$$


and the result holds by (4.23) for $\alpha=1$ and $\gamma \leqslant-2$. For $\sigma=2$ we need only to replace $|\ln h|^{2 n-1}$ by $|\ln h|^{2 n}$ and if $\sigma>2$, then $\omega(\varphi, h) \leqslant$ const $h$, and the result follows in a very similar fashion.

(ii)(b) Since in this case $y \in C^{4}(0,1]$, we can differentiate (3.1a) and obtain by [16, Lemma 3.3]

$$
\left|L_{j}\left(y_{\Delta}-R_{\Delta} y\right)\right| \leqslant \begin{cases}\text { const } h^{2} t_{j}^{\sigma-4}|\ln h|^{2 n-1}, & 0<\sigma<2, \\ \operatorname{const} h^{2} t_{j}^{-2}|\ln h|^{2 n} & \sigma=2, \quad i=1(1) N, \\ \text { const } h^{2} t_{j}^{-2}, & \sigma>2 .\end{cases}
$$

The estimates in (ii)(b) hold now by (4.23), on noting that in this case $y_{N}^{\prime}-y^{\prime}(1)=$ $O\left(h^{2}\right)$.

We shall now extend the results of Lemmas 4.1-4.3 to the general case where $J$ consists of some Jordan boxes with different eigenvalues.

LEMMA 4.4. For each $t_{i_{0}}=\varepsilon>0$, there exists an $h(\varepsilon)$ such that, for each $h<h(\varepsilon)$, the system (4.11) has a unique solution for every $Q u_{i_{0}}=\delta_{0}, P u_{N}=\delta_{N}, f_{\Delta}$ and furthermore

(i) $\left\|u_{\Delta}\right\| \leqslant$ const $\left\{\left|\delta_{0}\right|\left\|\left.\ln h\right|^{d_{0}-1}+\left|\delta_{N}\right|+\right\| f_{\Delta} \|\right\}$,

(ii) Let $y_{\Delta}$ be a solution of (4.1a).

(a) If $f \in C$, then

$$
\left\|y_{\Delta}-R_{\Delta} y\right\| \leqslant \begin{cases}\operatorname{const}\left\{h^{\sigma_{+}}|\ln h|^{d_{+}-1}+\omega(f, h)\right\}, & 0<\sigma_{+} \leqslant 1, \\ \operatorname{const}\left\{h|\ln h|^{d_{+}-1}+\omega(f, h)\right\}, & 1<\sigma_{+}<2, \\ \operatorname{const}\left\{h|\ln h|^{d_{+}}+\omega(f, h)\right\}, & \sigma_{+}=2, \\ \operatorname{const}\{h+\omega(f, h)\}, & \sigma_{+}>2 \text { or } S=0 .\end{cases}
$$

(b) If $f \in C^{2}$, then

$$
\left\|y_{\Delta}-R_{\Delta} y\right\| \leqslant \begin{cases}\text { const } h^{\sigma_{+}}|\ln h|^{d_{+}-1}, & 0<\sigma_{+}<2, \\ \text { const } h^{2}\left(|\ln h|^{d_{+}}+|\ln h|^{d_{0}-1}\right), & \sigma_{+}=2, \\ \text { const } h^{2}|\ln h|^{d_{0}-1}, & \sigma_{+}>2 \text { or } S=0 .\end{cases}
$$

Proof. (i) Let $M=E J E^{-1}$, where $J$ is the Jordan canonical form of $M$. Then the result follows as in Lemmas 4.1, 4.2 and 4.3 on considering the encoupled system corresponding to (4.13) first and using the contraction argument afterwards.

(ii) This follows from previous lemmas and (i).

Remark. (i) The results (ii) of Lemma 4.4 can be improved when the eigenvalues $\lambda=0$ of $M$ are simple.

(i)(a) Consider the system (4.13) and $v_{i_{0}}=\delta_{0}$. Then it follows immediately that

$$
\left|v_{i}\right| \leqslant \begin{cases}\operatorname{const}\left\{\left|\delta_{0}\right|+h^{\alpha} t_{i}^{\gamma+2}\left\|r_{\Delta}\right\|\right\}, & \gamma>-2 \\ \operatorname{const}\left\{\left|\delta_{0}\right|+h^{\alpha}\left|\ln t_{i_{0}}\right|\left\|r_{\Delta}\right\|\right\}, & \gamma=-2 \\ \operatorname{const}\left\{\left|\delta_{0}\right|+h^{\alpha} t_{i_{0}}^{\gamma+2}\left\|r_{\Delta}\right\|\right\}, & \gamma<-2\end{cases}
$$


(i)(b) For $v_{N}=\delta_{1}$ we have

$$
\left|v_{i}\right| \leqslant \begin{cases}\operatorname{const}\left\{\left|\delta_{1}\right|+h^{\alpha}\left\|r_{\Delta}\right\|\right\}, & \gamma>-2, \\ \operatorname{const}\left\{\left|\delta_{1}\right|+h^{\alpha}\left|\ln t_{i}\right|\left\|r_{\Delta}\right\|\right\}, & \gamma=-2, \\ \operatorname{const}\left\{\left|\delta_{1}\right|+h^{\alpha} t_{i}^{\gamma+2}\left\|r_{\Delta}\right\|\right\}, & \gamma<-2 .\end{cases}
$$

Finally, it follows from Lemma 4.4, (ii)(b), that for the case when (4.33a) holds

$$
\left\|y_{\Delta}-R_{\Delta} y\right\| \leqslant \text { const } h^{2}, \quad \text { if } \sigma_{+}>2 \text { or } S=0 .
$$

(ii) We shall now extend the results of Lemma 4.4 to problem (4.1). We first assume that the associated analytical problem (3.1) has a unique solution $y \in C \cap C^{2}(0,1]$ and recall that the necessary condition for the solution $y$ of (3.1) to be continuous is given by $\tilde{Q} Y(0)=0$. To show that the results of Lemma 4.4 hold also for the solution of (4.1) we have to consider two problems:

(ii)(a) We have to prove that the solution $u_{\Delta}$ of the difference equation (4.11) subject to the boundary conditions $Q u_{0}=0, P u_{N}=\delta_{N}$ yields the solution $y_{\Delta}$ of the difference equation (4.1a) subject to the boundary conditions (4.1b) and (4.1c).

Before proceeding further we briefly recapitulate how we have treated the analogous problem in the analytical case; cf. [16, Section 3]. First, we have considered the three cases where $\sigma<0, \lambda=0$, and $\sigma>0$, separately, and in each case we have given the conditions that are necessary for the solution to be continuous and the conditions that are required to make the solution unique. Using the spectral projections, we have constructed the general solution (for the case when $M$ has different eigenvalues) by superposition of continuous components pertaining to different eigenvalues. We have shown that the condition $Q u(0)=0$ is necessary for $u$ to be in $C[0,1]$ and the condition $P u(1)=P \gamma$ yields to $m=\operatorname{rank}[P]$ equations for the constants that have to be specified to make the solution unique. This solution has the form

$$
z(t)=(H f)(t)+\Phi(t) P \gamma
$$

where

$$
\begin{gathered}
(H f)(t)=t^{2} \int_{0}^{1} Q s^{-M^{M}} s f(t s) d s+t^{M} \int_{1}^{t} P s^{-M} s f(s) d s, \\
\Phi(t)=t^{M} P .
\end{gathered}
$$

Clearly, for problem (1.1a) the $m$ conditions which we need for $y$ to be unique have to be given by (1.1b). It has been shown in [16, Theorem 3.1] that the $m$ constants can be uniquely determined from (1.1b) if and only if the inverse of a certain $m \times m$ matrix which we obtain by substituting the solution $y(t)=z_{1}(t)$ and its derivative into (1.1b) exists.

We can use this idea again in the case of difference equations. Comparing the representations for the analytical and discrete solutions in each of the three cases mentioned above, and, finally, in the general case, we can show that the solution of the difference system can be written in a way which is a discrete analogue of that of the analytical case. To see this we define

$$
R_{\Delta}^{0} f=(f(0), f(h), f(2 h), \ldots, f(i h), \ldots, f(1)),
$$


and then the solution $u_{\Delta}$ of $(4.11)$ can be written as

$$
u_{\Delta}=H_{\Delta}\left(R_{\Delta}^{0} f\right)+\Phi_{\Delta} P u_{N} .
$$

This form corresponds to that given above. Substituting this general solution into the boundary conditions, we obtain a system of equations for $m$ constants that uniquely determines the discrete solution of (4.1). This system is uniquely solvable if and only if the same $m \times m$ matrix as in the analytical case is nonsingular.

(ii)(b) The second problem is how to pose the boundary conditions at the left point of the interval if $i_{0} \geqslant 1$.

We have chosen

$$
\begin{gathered}
B_{0}\left[\begin{array}{c}
y_{i_{0}} \\
\left(y_{i_{0}+1}-y_{i_{0}-1}\right) / 2 h
\end{array}\right]+B_{1}\left[\begin{array}{c}
y_{N} \\
\left(y_{N+1}-y_{N-1}\right) / 2 h
\end{array}\right]=\beta, \\
\tilde{Q}\left[\begin{array}{c}
y_{i_{0}} \\
\left(y_{i_{0}+1}-y_{i_{0}-1}\right) / 2 h
\end{array}\right]=\tilde{Q} Y(0)=0,
\end{gathered}
$$

and it follows by Lemma 4.4 and Remark (ii)(a) that the estimate (i) and the convergence rates from Lemma 4.4 remain valid. Clearly, we have to replace $\delta_{N}$ by $\beta$ in (i).

For the practical computations we have chosen $i_{0}=0$ and modified the boundary conditions as follows

$$
\begin{gathered}
B_{0}\left[\begin{array}{c}
y_{0} \\
\left(y_{1}-y_{0}\right) / h-h A^{-1} f(0) / 2
\end{array}\right]+B_{1}\left[\begin{array}{c}
y_{N} \\
\left(y_{N+1}-y_{N-1}\right) / 2 h
\end{array}\right]=\beta, \\
\tilde{Q}\left[\begin{array}{c}
y_{0} \\
\left(y_{1}-y_{0}\right) / h-h A^{-1} f(0) / 2
\end{array}\right]=0,
\end{gathered}
$$

where the matrix $A$ is defined as at the beginning of Section 4 . We can motivate the choice of $i_{0}=0$ by the fact that the restriction on $i_{0}$ following from Lemma 4.4 is sufficient (but not necessary) for the existence of the operator $(I-F)^{-1}$, where $F$ is the Fréchet derivative of $G$. The choice of $i_{0}=0$ worked well also for some problems with variable coefficient matrices $A_{0}(t)$ and $A_{1}(t)$ as well as for nonlinear examples. This could suggest that the theoretical results presented here can be extended to the whole interval $[0,1]$. This may be the subject of further considerations.

The extension of the theoretical results to the boundary conditions (4.35) is obvious.

We now consider the linear system (4.1a) with

$$
A_{i}(t)=A_{i}+t^{\nu} C_{i}(t), \quad i=0,1,
$$

where $\nu \geqslant 1$ and $C_{i}(t) \in C[0,1]$. To show the existence and the uniqueness of the solution of (4.1) we use contraction again. First of all we shall derive an analogous equation to (4.11) for this case. For $A_{i}(t)$ given by (4.36) we have

$$
\Theta_{1}(i, h)=\left(I-\frac{A_{1}+t_{i}^{\nu} C_{1}\left(t_{i}\right)}{2 i}\right)^{-1}\left(I+\frac{A_{1}\left(t_{i}\right)}{2}\right) A_{0}\left(t_{i}\right),
$$

$$
\Theta_{2}(i, h)=\left(I-\frac{A_{1}+t_{i}^{\nu} C_{1}\left(t_{i}\right)}{2 i}\right)^{-1}\left[\left(1+\frac{1}{i}\right) A_{0}\left(t_{i}\right)+\left(I+\frac{A_{1}\left(t_{i}\right)}{2}\right) A_{1}\left(t_{i}\right)\right] \text {, }
$$




$$
\Theta_{3}(i, h)=\left(I-\frac{A_{1}+t_{i}^{\nu} C_{1}\left(t_{i}\right)}{2 i}\right)^{-1}
$$

and we assume that for $1 \leqslant i \leqslant \max _{0 \leqslant t \leqslant 1}\left(\left\|A_{1}(t)\right\| / 2\right), \Theta_{j}(i, h), j=1,2,3$, exist. Then we have the following system for $u_{i}$

$$
u_{i}=u_{i-1}+\frac{1}{i} M\left(t_{i}\right) u_{i-1}+\frac{1}{i^{2}} \Theta_{4}(i, h) u_{i-1}+t_{i+1} h \Theta_{5}(i, h) \dot{f}_{i},
$$

where

$$
\Theta_{4}(i, h)=\left[\begin{array}{cc}
0 & 0 \\
\Theta_{1}(i, h) & \Theta_{2}(i, h)
\end{array}\right], \quad \Theta_{5}(i, h)=\left[\begin{array}{cc}
0 & 0 \\
0 & \Theta_{3}(i, h)
\end{array}\right]
$$

and

$$
M\left(t_{i}\right)=M+t_{i}^{\nu} \stackrel{\circ}{C}\left(t_{i}\right), \quad \stackrel{\circ}{C}\left(t_{i}\right)=\left[\begin{array}{cc}
0 & 0 \\
C_{0}\left(t_{i}\right) & C_{1}\left(t_{i}\right)
\end{array}\right] .
$$

We rewrite (4.38) and obtain

$$
\begin{aligned}
u_{i}= & u_{i-1}+\frac{1}{i} M u_{i-1}+\frac{t_{i}^{v}}{i} \dot{C}\left(t_{i}\right) u_{i-1}+\frac{1}{i^{2}} \Theta_{4}(i, h) u_{i-1} \\
& +t_{i+1} \Theta_{5}(i, h) \dot{f}_{i}, \quad i=\left(i_{0}+1\right)(1) N_{0},
\end{aligned}
$$

subject to the boundary conditions

$$
Q u_{i_{0}}=\delta_{0}, \quad P u_{N_{0}}=\delta_{N} .
$$

Note that the boundary value problem (4.39) is defined on the interval $\left[t_{i_{0}}, t_{N_{0}}\right]$, where $t_{i_{0}}=\varepsilon>0$ and $t_{N_{0}}=\tau \leqslant 1$. We consider the iteration scheme, as defined in (4.24) applied to (4.39) and show that $G$ is contracting if $t_{N_{0}}$ is small enough. The proof of this statement can be shown by consideration of the three previous cases separately and the subsequent extension to the general case. Since the arguments used are very similar, we shall not repeat the whole analysis but just derive the conditions which are sufficient for $G$ to be contracting.

If $\sigma<0$, then

$$
\left|G_{i} V-G_{i} W\right| \leqslant C \cdot\left\{\frac{h}{t_{i_{0}}}\left\|\Theta_{\Delta}\right\|+t_{i+1}^{\nu}\left\|\dot{C}_{\Delta}\right\|\right\}\left\|(V-W)_{\Delta}\right\|
$$

and hence

$$
\left\|(G V-G W)_{\Delta}\right\| \leqslant C\left\{\frac{h}{t_{i_{0}}}\left\|\Theta_{\Delta}\right\|+t_{N_{0}+1}^{\nu}\left\|\dot{C}_{\Delta}\right\|\right\}\left\|(V-W)_{\Delta}\right\| .
$$

If $\lambda=0$, then from (4.27)

$$
\left\|(G V-G W)_{\Delta}\right\| \leqslant C\left\{\frac{h}{t_{i_{0}}}\left|\ln t_{i_{0}}\right|^{2 n-1}\left\|\Theta_{\Delta}\right\|+t_{N_{0}+1}^{\nu}\left\|\dot{C}_{\Delta}\right\|\right\}\left\|(V-W)_{\Delta}\right\| .
$$

Let $\sigma>0$. Then we have

$$
\left\|(G V-G W)_{\Delta}\right\| \leqslant C\left\{\frac{h}{t_{i_{0}}}\left\|\Theta_{\Delta}\right\|+t_{N_{0}+1}^{\nu}\left\|\dot{C}_{\Delta}\right\|\right\}\left\|(V-W)_{\Delta}\right\| .
$$


Let $t_{i_{0}}=\varepsilon>0$. Then $\left\|(G V-G W)_{\Delta}\right\|<\left\|(V-W)_{\Delta}\right\|$ if $h$ and $t_{N_{0}}$ are sufficiently small. The standard contraction argument yields now the existence and uniqueness of the solution of (4.39) on $[\varepsilon, \tau]$, where $\tau \leqslant 1$. It is clear from (4.39a) that this solution can be uniquely continued to $\tau=1$, and the following lemma is obvious; see also [16, Theorem 4.2] and Remark (ii).

The convergence rates in part (ii) have been obtained as in Lemma 4.4, using the smoothness properties of $y$ from Section 3.

LEMMA 4.5. If the homogeneous boundary value problem (3.1) has only the trivial solution, then, for each $t_{i_{0}}=\varepsilon>0$, there exists an $h(\varepsilon)>0$ such that, for every $h \leqslant h(\varepsilon)$, the system (4.1a) subject to the boundary conditions

$$
B_{0} Y_{i_{0}}+B_{1} Y_{N}=\beta, \quad \tilde{Q} Y_{i_{0}}=\delta_{0},
$$

has a unique solution for each $f_{\Delta}$ and the following estimate holds:

(i) $\left\|y_{\Delta}\right\| \leqslant$ const $\left\{\left|\delta_{0}\right||\ln h|^{d_{0}-1}+|\beta|+\| f_{\Delta}||\right.$.

(ii) Let $y_{\Delta}$ be a solution of (4.1a) subject to the boundary conditions (4.43) with $\delta_{0}=0$.

(a) If $f, C_{1} \in C$ and $C_{0} \in C^{1}$, then

$$
\left\|y_{\Delta}-R_{\Delta} y\right\| \leqslant \begin{cases}\operatorname{const}\left\{h^{\sigma_{+}}|\ln h|^{d_{+}-1}+\omega(f, h)\right\}, & 0<\sigma_{+} \leqslant 1, \\ \operatorname{const}\left\{h|\ln h|^{d_{+}-1}+\omega(f, h)\right\}, & 1<\sigma_{+}<2, \\ \operatorname{const}\left\{h|\ln h|^{d_{+}}+\omega(f, h)\right\}, & \sigma_{+}=2, \\ \operatorname{const}\{h+\omega(f, h)\}, & \sigma_{+}>2 \text { or } S=0 .\end{cases}
$$

(b) Iff, $C_{1} \in C^{2}$ and $C_{0} \in C^{3}$, then

$$
\left\|y_{\Delta}-R_{\Delta} y\right\| \leqslant \begin{cases}\text { const } h^{\sigma_{+}}|\ln h|^{d_{+}-1}, & 0<\sigma_{+}<2, \\ \text { const } h^{2}\left(|\ln h|^{d_{+}}+|\ln h|^{d_{0}-1}\right), & \sigma_{+}=2, \\ \text { const } h^{2}|\ln h|^{d_{0}-1}, & \sigma_{+}>2 \text { or } S=0 .\end{cases}
$$

\section{Numerical Examples.}

Example 1. We consider the scalar equation

$$
y^{\prime \prime}(t)-\frac{(\sqrt{5}-1)}{t} y^{\prime}(t)-\frac{1}{t^{2}} y(t)=3-2 \sqrt{5} \text {. }
$$

There are two different eigenvalues

$$
\lambda_{1}=(\sqrt{5}-3) / 2<0, \quad \lambda_{2}=(\sqrt{5}+3) / 2>2,
$$

and therefore we choose the following boundary conditions

$$
y(0)=0, \quad y(1)=0 .
$$

This yields the continuous solution of the form

$$
y(t)=t^{2}-t^{(\sqrt{5}+3) / 2},
$$

and we expect that the error behave like $h^{2}$. 
Example 2. We have now the following scalar problem

$$
y^{\prime \prime}(t)-\frac{0.5}{t} y^{\prime}(t)=-1, \quad y(0)=y(1)=5,
$$

whose solution is

$$
y(t)=5-t^{2}+t^{3 / 2} .
$$

The convergence rate of $h^{3 / 2}$ is illustrated in Table 5.1, where the corresponding results for the first example are also tabulated. For both examples $i_{0}=0$.

TABLE 5.1

\begin{tabular}{c|c|c||c|c}
\hline & \multicolumn{2}{|c||}{ Example 1 } & \multicolumn{2}{c}{ Example 2 } \\
\hline \multicolumn{1}{c|}{$h$} & $\Delta=\left|y_{\Delta}-R_{\Delta .} y\right|$ & $\Delta / h^{2}$ & $\Delta=\left|y_{\lrcorner}-R_{\lrcorner} .{ }^{\prime}\right|$ & $\Delta / h^{3 / 2}$ \\
\hline $1 / 10$ & $1.480 \mathrm{E}-3$ & 0.1480 & $3.647 \mathrm{E}-3$ & 0.1153 \\
$1 / 20$ & $3.748 \mathrm{E}-4$ & 0.1499 & $1.462 \mathrm{E}-3$ & 0.1308 \\
$1 / 40$ & $9.437 \mathrm{E}-5$ & 0.1510 & $5.650 \mathrm{E}-4$ & 0.1429 \\
$1 / 80$ & $2.370 \mathrm{E}-5$ & 0.1517 & $2.130 \mathrm{E}-4$ & 0.1524 \\
$1 / 160$ & $5.935 \mathrm{E}-6$ & 0.1519 & $7.894 \mathrm{E}-5$ & 0.1598 \\
$1 / 320$ & $1.485 \mathrm{E}-6$ & 0.1521 & $2.891 \mathrm{E}-5$ & 0.1655 \\
$1 / 640$ & $3.715 \mathrm{E}-7$ & 0.1520 & $1.049 \mathrm{E}-5$ & 0.1698 \\
\hline
\end{tabular}

Example 3. We now consider the $2 \times 2$ system (3.1a) where

$$
A_{1}=\left[\begin{array}{cc}
-1 & 1 \\
-9 / 4 & -4
\end{array}\right], \quad A_{0}=\left[\begin{array}{ll}
1 & 0 \\
0 & 1
\end{array}\right], \quad f(t)=\left[\begin{array}{c}
6-3 t \\
9+17 t
\end{array}\right] .
$$

The Jordan canonical form of $M$ is

$$
J=\left[\begin{array}{rccc}
-2 & 1 & 0 & 0 \\
0 & -2 & 0 & 0 \\
0 & 0 & 1 / 2 & 1 \\
0 & 0 & 0 & 1 / 2
\end{array}\right],
$$

and hence $y(0)=0$ is the necessary condition for $y$ to be continuous. Furthermore we choose

$$
\text { (a) } y(1)+y^{\prime}(1)=\left[\begin{array}{l}
6 \\
4
\end{array}\right], \quad \text { (b) } y(1)+y^{\prime}(1)=\left[\begin{array}{r}
-12 \\
31
\end{array}\right] \text {, }
$$

and the corresponding solutions are

$$
y^{a}(t)=\left[\begin{array}{c}
2 t^{2} \\
t^{3}
\end{array}\right], \quad y^{b}(t)=\left[\begin{array}{c}
-12 \sqrt{t}+2 t^{2} \\
18 \sqrt{t}+t^{3}
\end{array}\right] .
$$

Since the error behavior does not depend on the component of these solutions, we list in the following tables the global error in the first component and the error at a fixed point $t=0.5$ in the second component of each solution. In both cases $i_{0}=0$. 
TABLE 5.2

Example 3(a)

\begin{tabular}{l|c|c|c|c}
\hline \multicolumn{1}{c|}{$h$} & $\begin{array}{c}\text { lst component } \\
\Delta_{1}\end{array}$ & $\Delta_{1} / h^{2}$ & $\begin{array}{c}\text { 2nd component } \\
\Delta_{2}\end{array}$ & $\Delta_{2} / h^{2}$ \\
\hline $1 / 4$ & $1.374 \mathrm{E}-2$ & 0.219 & $2.905 \mathrm{E}-2$ & 0.465 \\
$1 / 8$ & $3.336 \mathrm{E}-3$ & 0.214 & $6.686 \mathrm{E}-3$ & 0.428 \\
$1 / 16$ & $7.721 \mathrm{E}-4$ & 0.198 & $1.647 \mathrm{E}-3$ & 0.422 \\
$1 / 32$ & $1.711 \mathrm{E}-4$ & 0.175 & $4.105 \mathrm{E}-4$ & 0.420 \\
$1 / 64$ & $3.667 \mathrm{E}-5$ & 0.150 & $1.026 \mathrm{E}-4$ & 0.420 \\
$1 / 128$ & $9.111 \mathrm{E}-6$ & 0.149 & $2.564 \mathrm{E}-5$ & 0.420 \\
\hline
\end{tabular}

TABLE 5.3

Example 3(b)

\begin{tabular}{l|c|c|c|c}
\multicolumn{1}{c|}{$h$} & $\begin{array}{c}\text { 1st component } \\
\Delta_{1}\end{array}$ & $\Delta_{1} / \sqrt{h}$ & $\begin{array}{c}\text { 2nd component } \\
\Delta_{2}\end{array}$ & $\Delta_{2} / h^{2}$ \\
\hline $1 / 4$ & $4.996 \mathrm{E}-1$ & 0.999 & $2.729 \mathrm{E}-1$ & 4.37 \\
$1 / 8$ & $3.484 \mathrm{E}-1$ & 0.985 & $6.616 \mathrm{E}-2$ & 4.23 \\
$1 / 16$ & $2.453 \mathrm{E}-1$ & 0.981 & $1.643 \mathrm{E}-2$ & 4.21 \\
$1 / 32$ & $1.732 \mathrm{E}-1$ & 0.980 & $4.102 \mathrm{E}-3$ & 4.20 \\
$1 / 64$ & $1.224 \mathrm{E}-1$ & 0.979 & $1.025 \mathrm{E}-3$ & 4.19 \\
$1 / 128$ & $8.652 \mathrm{E}-2$ & 0.978 & $2.563 \mathrm{E}-4$ & 4.19 \\
\hline
\end{tabular}

Example 4. To illustrate the results of Lemma 4.5 we investigate the $2 \times 2$ system with

$$
\begin{aligned}
& A_{1}=\left[\begin{array}{rr}
-1 & 0 \\
0 & -1
\end{array}\right], \quad A_{0}=\left[\begin{array}{cc}
0 & 0 \\
0 & 1 / 4
\end{array}\right], \\
& f(t)=\left[\begin{array}{c}
y_{2}(t) \\
100 y_{1}(t)-100 x^{5}+200 x^{2.5}+218.75 x-100
\end{array}\right]
\end{aligned}
$$

and the boundary conditions

$$
y_{1}^{\prime}(0)=0, \quad y_{2}(0)=0 ; \quad y_{1}(1)=0, \quad y_{1}^{\prime}(1)=0 .
$$

The corresponding eigenvalues of $M$ are $\lambda_{1}=\lambda_{2}=0, \lambda_{3}=1 / 2, \lambda_{4}=-1 / 2$ and the Jordan canonical form is

$$
J=\left[\begin{array}{cccc}
1 / 2 & 0 & 0 & 0 \\
0 & -1 / 2 & 0 & 0 \\
0 & 0 & 0 & 1 \\
0 & 0 & 0 & 0
\end{array}\right]
$$

Since the problem has a solution $y \in C \cap C^{1}(0,1]$, it follows from Lemma 4.5(ii) that the convergence rate is $O(\sqrt{h})$. 
TABLE 5.4

\begin{tabular}{c|c|c|c}
\hline$h$ & $\Delta(h)=\left\|y_{\Delta}-R_{\Delta} y\right\|$ & $\Delta(h) / \sqrt{h}$ & $\Delta(h / 2) / \Delta(h)$ \\
\hline $1 / 10$ & $3.044 \mathrm{E}-1$ & 0.9626 & $*$ \\
$1 / 20$ & $3.143 \mathrm{E}-1$ & 1.4056 & 1.0325 \\
$1 / 40$ & $2.320 \mathrm{E}-1$ & 1.4673 & 0.7381 \\
$1 / 80$ & $1.630 \mathrm{E}-1$ & 1.4579 & 0.7026 \\
$1 / 160$ & $1.141 \mathrm{E}-1$ & 1.4433 & 0.7000 \\
$1 / 320$ & $8.014 \mathrm{E}-2$ & 1.4336 & 0.7024 \\
$1 / 640$ & $5.645 \mathrm{E}-2$ & 1.4281 & 0.7044 \\
\hline
\end{tabular}

$$
\lim _{h \rightarrow 0} \frac{\Delta(h / 2)}{\Delta(h)}=0.7071
$$

Example 5. The next system is two-dimensional and

$$
A_{1}=\left[\begin{array}{cc}
4 & 0 \\
1 & -3
\end{array}\right], \quad A_{0}=\left[\begin{array}{cc}
-25 / 4 & 0 \\
0 & 0
\end{array}\right], \quad f=\left[\begin{array}{r}
1 \\
-8
\end{array}\right] \text {. }
$$

$M$ has the following eigenvalues $\lambda_{1}=-2, \lambda_{2}=0, \lambda_{3}=\lambda_{4}=2.5$. The boundary conditions are

$$
y_{2}(0)=3, \quad y_{2}^{\prime}(0)=0 ; \quad y_{1}(1)=8, \quad y_{1}^{\prime}(1)=36,
$$

and the continuous solution of this problem is

$$
y(t)=\left[\begin{array}{c}
18 t^{2.5} \ln t+4 t^{2.5}+4 t^{2} \\
3+4 t^{2.5} \ln t
\end{array}\right]
$$

From the last remark we expect the convergence to be of second order and this is verified by the results from the next table.

\begin{tabular}{c|c|c}
\multicolumn{3}{c}{ TABLE 5.5} \\
\hline$h$ & $\Delta=\left\|y_{\Delta}-R_{\Delta} y\right\|$ & $\Delta / h^{2}$ \\
\hline $1 / 10$ & $7.502 \mathrm{E}-2$ & 7.502 \\
$1 / 20$ & $1.861 \mathrm{E}-2$ & 7.444 \\
$1 / 40$ & $4.645 \mathrm{E}-3$ & 7.432 \\
$1 / 80$ & $1.161 \mathrm{E}-3$ & 7.430 \\
$1 / 160$ & $2.903 \mathrm{E}-4$ & 7.432 \\
$1 / 320$ & $7.257 \mathrm{E}-5$ & 7.431 \\
\hline
\end{tabular}

Example 6. Finally, we consider the following homogeneous system to illustrate the results of Lemma 4.4 (ii)(b) in the case when $J$ consists of two Jordan boxes. Let

$$
A_{1}=\left[\begin{array}{ll}
a_{1} & 0 \\
0 & a_{1}
\end{array}\right], \quad A_{0}=\left[\begin{array}{ll}
0 & a_{0} \\
0 & 0
\end{array}\right], \quad f(t)=\left[\begin{array}{l}
0 \\
0
\end{array}\right] .
$$

If $a_{1}=0.5, a_{0}=1$, then $\lambda_{1}=\lambda_{2}=0, \lambda_{3}=\lambda_{4}=1.5$ and the boundary conditions

$$
y_{1}(0)=3, \quad y_{2}(0)=0 ; \quad y_{1}^{\prime}(1)=-1, \quad y_{2}(1)=3
$$

yield the solution

$$
y(t)=\left[\begin{array}{c}
3-2 t^{1.5}(1-\ln t) \\
3 t^{1.5}
\end{array}\right]
$$


If $a_{1}=-0.2, a_{0}=1$, then $\lambda_{1}=\lambda_{2}=0, \lambda_{3}=\lambda_{4}=0.8$ and if

$$
y_{1}(0)=3, \quad y_{2}(0)=0 ; \quad y_{1}^{\prime}(1)=0.4, \quad y_{2}(1)=1.6 \text {, }
$$

then the solution is

$$
y(t)=\left[\begin{array}{c}
3-2 t^{0.8}(1-\ln t) \\
1.6 t^{0.8}
\end{array}\right] .
$$

The error behavior follows by Lemma 4.4 on noting that there are no logarithmic terms in the second components of both solutions $y(t)$.

TABLE 5.6 (i)

\begin{tabular}{c|c|c|c||c|c|c}
\hline & \multicolumn{3}{|c||}{ Ist component } & \multicolumn{3}{c}{ 2nd component } \\
\hline$h$ & $\Delta(h)$ & $\Delta(h) / h^{1.5}|\ln h|$ & $\Delta(h / 2) / \Delta(h)$ & $\Delta(h)$ & $\Delta(h) / h^{1.5}$ & $\Delta(h / 2) / \Delta(h)$ \\
\hline $1 / 10$ & $4.751 \mathrm{E}-2$ & 0.6525 & $*$ & $1.094 \mathrm{E}-2$ & 0.3460 & $*$ \\
$1 / 20$ & $2.185 \mathrm{E}-2$ & 0.6524 & 0.460 & $4.385 \mathrm{E}-3$ & 0.3922 & 0.401 \\
$1 / 40$ & $9.575 \mathrm{E}-3$ & 0.6567 & 0.440 & $1.695 \mathrm{E}-3$ & 0.4288 & 0.387 \\
$1 / 80$ & $4.057 \mathrm{E}-3$ & 0.6625 & 0.424 & $6.390 \mathrm{E}-4$ & 0.4572 & 0.377 \\
$1 / 160$ & $1.677 \mathrm{E}-3$ & 0.6687 & 0.413 & $2.368 \mathrm{E}-4$ & 0.4792 & 0.371 \\
$1 / 320$ & $6.795 \mathrm{E}-4$ & 0.6743 & 0.405 & $8.672 \mathrm{E}-5$ & 0.4964 & 0.366 \\
$1 / 640$ & $2.712 \mathrm{E}-4$ & 0.6796 & 0.400 & $3.148 \mathrm{E}-5$ & 0.5097 & 0.363 \\
\hline
\end{tabular}

$$
\lim _{h \rightarrow 0} \Delta(h / 2) / \Delta(h)=(1 / 2)^{1.5} \approx 0.354
$$

TABLE 5.6 (ii)

\begin{tabular}{c|c|c|c||c|c|c}
\hline & \multicolumn{3}{|c||}{ Ist component } & \multicolumn{3}{c}{ 2nd component } \\
\hline$h$ & $\Delta(h)$ & $\Delta(h) / h^{0.8}|\ln h|$ & $\Delta(h / 2) / \Delta(h)$ & $\Delta(h)$ & $\Delta(h) / h^{0.8}$ & $\Delta(h / 2) / \Delta(h)$ \\
\hline $1 / 10$ & $3.809 \mathrm{E}-1$ & 1.0437 & $*$ & $2.161 \mathrm{E}-2$ & 0.1363 & $*$ \\
$1 / 20$ & $2.450 \mathrm{E}-1$ & 0.8984 & 0.643 & $1.342 \mathrm{E}-2$ & 0.1474 & 0.621 \\
$1 / 40$ & $1.562 \mathrm{E}-1$ & 0.8099 & 0.638 & $8.037 \mathrm{E}-3$ & 0.1537 & 0.600 \\
$1 / 80$ & $9.884 \mathrm{E}-2$ & 0.7512 & 0.633 & $4.887 \mathrm{E}-3$ & 0.1627 & 0.608 \\
$1 / 160$ & $6.205 \mathrm{E}-2$ & 0.7089 & 0.628 & $2.889 \mathrm{E}-3$ & 0.1675 & 0.591 \\
$1 / 320$ & $3.869 \mathrm{E}-2$ & 0.6771 & 0.623 & $1.693 \mathrm{E}-3$ & 0.1709 & 0.586 \\
$1 / 640$ & $2.398 \mathrm{E}-2$ & 0.6523 & 0.620 & $9.863 \mathrm{E}-4$ & 0.1734 & 0.583 \\
\hline
\end{tabular}

$$
\lim _{h \rightarrow 0} \Delta(h / 2) / \Delta(h)=(1 / 2)^{0.8} \approx 0.574
$$

All experiments have been carried out on a CDC Cyber 170/720 in single precision (48 bit mantissa).

Institut für Angewandte und Numerische Mathematik

Technische Universität Wien

Gusshausstrasse 27-29

A-1040 Wien, Austria

1. D. C. Brabston \& H. B. Keller, "A numerical method for singular two point boundary value problems," SIAM J. Numer. Anal., v. 14, 1977, pp. 779-791.

2. F. R. DE HOOG \& R. WEISS, "Difference methods for boundary value problems with a singularity of the first kind," SIAM J. Numer. Anal., v. 13, 1976, pp. 775-813. 
3. F. R. DE Hoog \& R. WeISs, "On the boundary value problem for systems of ordinary differential equations with a singularity of the second kind," SIAM J. Numer. Anal., v. 11, 1980, pp. 41-60.

4. N. Dunford \& J. T. SchwartZ, Linear Operators, Part I, Interscience, New York, 1967.

5. P. JAMET, "On the convergence of finite difference approximations to one-dimensional singular boundary-value problems," Numer. Math., v. 14, 1970, pp. 355-378.

6. T. Kato, Perturbation Theory for Linear Operators, Springer-Verlag, New York, 1966.

7. H. B. Keller \& A. W. Wolfe, "On the nonunique equilibrium states and buckling mechanism of spherical shells,” J. Soc. Indust. Appl. Math., v. 13, 1965, pp. 674-705.

8. H. B. KelleR, “Approximation methods for nonlinear problems with application to two-point boundary value problems," Math. Comp., v. 29, 1975, pp. 464-474.

9. Y. L. LuKE, Mathematical Functions and Their Approximations, Academic Press, New York, 1975.

10. F. NATTERER, "A generalized spline method for singular boundary value problems in ordinary differential equations," Linear Algebra Appl., v. 7, 1973, pp. 189-216.

11. F. NATTERER, "Das Differenzenverfahren für singuläre Rand-Eigenwertaufgaben gewöhnlicher Differentialgleichungen,” Numer. Math., v. 23, 1975, pp. 387-409.

12. S. V. Parter, M. L. Stein \& P. R. Stein, On the Multiplicity of Solutions of a Differential Equation Arising in Chemical Reactor Theory, Tech. Rep. 194, Dept. of Computer Sciences, Univ. of WisconsinMadison, 1973.

13. S. V. PARTER, A-Posteriori Error Estimates, Tech. Rep. 214, Dept. of Computer Sciences, Univ. of Wisconsin-Madison, 1974.

14. P. RentroP, Eine Taylorreihenmethode zur numerischen Lösung von Zwei-Punkt Randwertproblemen mit Anwendung auf singuläre Probleme der nichtlinearen Schalentheorie, TUM, Institut für Mathematik, München, 1977.

15. R. D. Russel \& L. F. Shampine, "Numerical methods for singular boundary value problems," SIAM J. Numer. Anal., v. 12, 1975, pp. 13-35.

16. E. WeINMÜLLER, "On the boundary value problem for systems of ordinary differential equations with a singularity of the first kind," SIAM J. Math. Anal., v. 15, 1984. (To appear.) 